\title{
Efficient magnetohydrodynamic simulations on distributed multi-GPU systems using a novel GPU Direct-MPI hybrid approach
}

\author{
Un-Hong Wong a,b \& Takayuki Aoki ${ }^{\mathrm{a}, \mathrm{b}}$ \& Hon-Cheng Wong ${ }^{\mathrm{c}, \mathrm{d}} 1$ \\ ${ }^{a}$ Department of Energy Sciences, \\ Interdisciplinary Graduate School of Science and Engineering, \\ Tokyo Institute of Technology, \\ 4259 Nagatsuta-cho, Midori-ku, Yokohama, Kanagawa 226-8502, Japan \\ ${ }^{\mathrm{b}}$ Global Scientific Information and Computing Center, \\ Tokyo Institute of Technology, \\ 2-12-1 Ookayama, Meguro-ku, Tokyo 152-8550, Japan \\ ${ }^{\mathrm{c}}$ Faculty of Information Technology, \\ Macau University of Science and Technology, Macao, China \\ d Space Science Institute, \\ Macau University of Science and Technology, Macao, China
}

\begin{abstract}
Modern graphics processing units (GPUs) have been widely utilized in magnetohydrodynamic (MHD) simulations in recent years. Due to the limited memory of a single GPU, distributed multi-GPU systems are needed to be explored for largescale MHD simulations. However, the data transfer between GPUs bottlenecks the efficiency of the simulations on such systems. In this paper we propose a novel GPU Direct-MPI hybrid approach to address this problem for overall performance enhancement. Our approach consists of two strategies: (1) We exploit GPU Direct 2.0 to speedup the data transfers between multiple GPUs in a single node and reduce the total number of message passing interface (MPI) communications; (2) We design Compute Unified Device Architecture (CUDA) kernels instead of using memory copy to speedup the fragmented data exchange in the three-dimensional (3D) decomposition. 3D decomposition is usually not preferable for distributed multi-GPU systems due to its low efficiency of the fragmented data exchange. Our approach has made a breakthrough to make 3D decomposition available on distributed multi-GPU systems. As a result, it can reduce the memory usage and computation time of each partition of the computational domain. Experiment results show twice the FLOPS comparing to common 2D decomposition MPI-only implementation method. The proposed approach has been developed in an efficient implementation for MHD simulations on distributed multi-GPU systems, called $M G P U-M H D$ code. The code
\end{abstract}

1 Corresponding author: Tel: +853 88972052, E-mail address: hcwong@ieee.org 
realizes the GPU parallelization of a total variation diminishing (TVD) algorithm 
for solving the multidimensional ideal MHD equations, extending our work for single GPU computation [H.-C. Wong, U.-H. Wong, X. Feng, Z. Tang, Efficient magnetohydrodynamic simulations on graphics processing units with CUDA, Computer Physics Communications. 182 (2011) 2132-2160] to multiple GPUs. Numerical tests and performance measurements are conducted on the TSUBAME 2.0 supercomputer at the Tokyo Institute of Technology. Our code achieves 2 TFLOPS in double precision for the problem with $1200^{3}$ grid points using 216 GPUs.

Key words: MHD simulations, GPUs, distributed multi-GPU systems, CUDA, Parallel computing

\section{Introduction}

High performance scientific computing using modern graphics processing units (GPUs) have been popularizing in the last few years since the advent of NVIDIA Compute Unified Device Architecture (CUDA) [14,20]. More and more numerical simulations in different fields have been performing on GPUs. To use GPUs for computations, data have to be stored in the video memory (GRAM) which is on the graphics board. But the capacity of GRAM on a single GPU is far from the requirement for large-scale simulations, thus distributed multi-GPU systems are needed. Nowadays, most of the supercomputers with high rankings in Top 500 list [37] contain GPUs to offer extremely high computation power, including Titan, Tianhe-1A, Nebulae, TSUBAME 2.0, and HA-PACS. Many-core GPUs provide very high floating point operations per second (FLOPS) and their power are kept increasing rapidly. The widely use of GPUs also drives their development, the architecture of GPUs is now being renewed in every two years, for example, the Kepler GPUs have about two times (in double precision) the peak FLOPS [22] of the Fermi GPUs [21], but the bandwidth does not increase as fast as the computation power. Moreover, data exchanges between GPUs have to go through the host memory (memory on the motherboard). This can cause extra overheads for computations using distributed multi-GPU systems. If the data are fragmented in the GRAM, the overheads will be quite heavy. That is why the common way of using distributed multi-GPU systems for large-scale scientific simulations is to decompose the whole computational domain in two-dimension (2D) (only do the partitioning along the $y$ and $z$ dimensions). On the other hand, data transfers between GPUs on distributed multi-GPU systems are also the bottleneck. Because the whole computational domain is decomposed into a certain number of partitions, and these partitions are stored and calculated in different noded, it is necessary to exchange the halo data of a partition with its neighboring partitions.

In recent years, an increasing number of work about plasma simulations (particle- 
in-cell (PIC) and MHD models) on GPUs have been presented and most of them work on a single GPU. The early work of Stantchev et al. [32,33] implemented a PIC code on GPUs for plasmas simulations and visualizations. The code demonstrated a speedup of 11-22 for different grid sizes on a NVIDIA GeForce 8800 Ultra graphics card. Abreu et al. [1] developed a 2D relativistic GPU PIC code and this code can perform simulations on a NVIDIA Tesla C1060 graphics card and direct visualizations on a NVIDIA Quadro FX 1800 graphics card. Kong et al. [15] developed a 2D3V fully relativistic electromagnetic code on a NVIDIA GeForce GTX 280 graphics card and achieved speedup of 81x and 27x over an Intel Core 2 Duo E7200 2.53 GHz CPU using only a single core for cold plasma runs and extremely relativistic plasma runs, respectively. Decyk and Singh [5] developed a new parameterized PIC algorithms and data structures on a NVIDIA GeForce GTX 280 graphics card. They reported speedups of about 15-25 compared to an Intel Nehalem $2.66 \mathrm{GHz}$ processor for a simple 2D electrostatic code. Madduri et al. [18] reported the first study on tuning gyrokinetic PIC algorithms for GPUs, using a NVIDIA C2050 graphics card. For multi-GPU implementations, Burau et al. [4] presented one of the first multi-GPU PIC implementation called PICon$G P U$, which is a fully relativistic, $2 \mathrm{D}$ electromagnetic code for simulating the acceleration of electrons in an under-dense plasma by a laser-driven wakefield. Bastrakov et al. [2] presented an approach to high-performance implementation of PIC algorithms on heterogeneous cluster systems.

There are also some implementations of MHD simulations on GPUs and most of them also work on a single GPU. Our previous work GPU-MHD code [43] implemented a total variation diminishing (TVD) algorithm on a NVIDIA GeForce GTX 290 graphics card and achieved a speedup of 84 times in 3D over an Intel Core i7 $9653.2 \mathrm{GHz}$ CPU. Pang et al. [25] also implemented the same algorithm on NVIDIA GTX 260 graphics card and reached a speedup of 105 times in 3D over a Xeon E5506 2.13 GHz CPU. Wang et al. [40] implemented a compressible inviscid fluid solvers and extended it to support MHD simulations on a NVIDIA Quadro FX 5600 graphics card. The code achieved a factor of ten speedup over a $3 \mathrm{GHz} \mathrm{CPU}$. They also tested the code on a small cluster with four nodes and each has a NVIDIA GeForce 8800 GT graphics card. They reported a close ideal speedup for up to four GPUs. Ueda et al. [39] implemented the CIP-MOCCT method on a NVIDIA GeForce GTX 480 graphics card and obtained speedups of 30 over a Intel i7 $2.93 \mathrm{GHz}$ CPU. Zink [47] developed a GPU-accelerated general relativistic MHD code HORIZON and tested it on a NVIDIA GeForce GTX 580 graphics card and two NVIDIA Tesla C2070 graphics cards installed in a workstation. Lin et al. [17] presented the large-scale high-Lundquist number reduced MHD simulations of the solar corona on GPU accelerated distributed memory machines. But a very limited of implementation details were reported in Lin's work. Recently, Wasiljew and Murawski [42] presented a GPU implementation of the 2D Athena code [35] and tested it on a NVIDIA GeForce GTX 460 graphics card. Our recent work 
[44] implemented a global MHD model on multiple GPUs on a single node, achieving a peak performance of 97.36 GFLOPS in double precision. Feng et al. [9] [10] performed MHD simulations on multiple GPUs. They present an OpenCL-based implementation of their Solar-Interplanetary-CESE MHD model (SIP-CESE MHD model) [8] on CPU/GPU clusters in double precision for studying the solar corona/interplanetary solar wind, achieving a performance speedup of $5 \times$ without any optimization compared to that of their CPU implementation. Although there are quite a number of GPU MHD implementations available, no optimization techniques dedicated to improve the performance of MHD simulations on distributed multi-GPU systems are presented in detail.

In this paper, we propose an efficient implementation of magnetohydrodynamic (MHD) simulations with optimization techniques on distributed multiGPU systems (called MGPU-MHD code) to tackle the data transfer bottleneck. GPU Direct $2.0[14,20]$ for peer-to-peer data transfer between GPUs has been used in our approach. The present work realizes the GPU parallelization of a TVD algorithm for solving the multidimensional ideal MHD equations, extending our previous work GPU-MHD code [43] for single GPU computation to multiple GPUs. MGPU-MHD code has been implemented and tested on the TSUBAME 2.0 supercomputer $[6,11]$ at the Tokyo Institute of Technology. TSUBAME 2.0 consists of 1,408 Hewlett-Packard Proliant SL390s nodes and each node is equipped with three NVIDIA Tesla M2050 (Fermi architecture) GPUs, thus the total number of GPUs is 4,224. Several interesting applications have been implemented on TSUBAME 2.0, for examples, ASUCA model for weather simulation [28], phase-field simulation for dendritic solidification [29,45], and lattice-Boltzmann based incompressible flow computation [41]. Most recently, Shimokawabe et al. [30] proposed a high-productivity framework for multi-GPU computation of mesh-based applications, utilizing peer-to-peer access between GPUs on TSUBAME 2.5. They performed diffusion equation computation using using two NVIDIA Tesla K20X GPUs on a same node in the framework, achieving 1.4 times faster than that of the manual implementation code.

The paper is organized as follows: A breif review of the ideal MHD equations and numerical method is given in Section 2. In Section 3 we highlight our previous work of MHD simulations on a single GPU. Extension of our single GPU MHD code and its efficient implementation on distributed multi-GPU systems are present in detail in Section 4. Numerical tests are given in Section 5. Performance measurements are reported in Section 6. We conclude our work and discuss some directions for possible future work in Section 7 . 


\section{Ideal MHD equations and numerical method}

In this section, we briefly review the ideal MHD equations and numerical method adopted in our implementation. For the detailed information about the numerical scheme, please refer to [43]. The ideal MHD equations with the assumption of the magnetic permeability $\mu=1$ can be expressed as hyperbolic system of conservation laws as follows [12]

$$
\begin{aligned}
\frac{\partial \rho}{\partial t}+\boldsymbol{\nabla} \cdot(\rho \boldsymbol{v}) & =0 \\
\frac{\partial \rho \boldsymbol{v}}{\partial t}+\boldsymbol{\nabla} \cdot(\rho \boldsymbol{v} \boldsymbol{v}-\boldsymbol{B} \boldsymbol{B})+\boldsymbol{\nabla} P^{*} & =0 \\
\frac{\partial \boldsymbol{B}}{\partial t}-\boldsymbol{\nabla} \times(\boldsymbol{v} \times \boldsymbol{B}) & =0 \\
\frac{\partial E}{\partial t}+\boldsymbol{\nabla} \cdot\left(\left(E+P^{*}\right) \boldsymbol{v}-\boldsymbol{B}(\boldsymbol{B} \cdot \boldsymbol{v})\right) & =0
\end{aligned}
$$

Here, $\rho$ is the mass density, $\rho \boldsymbol{v}$ the momentum density, $\boldsymbol{B}$ the magnetic field, and $E$ the total energy density. The total pressure $P^{*} \equiv P+\frac{B^{2}}{2}$ where $P$ is the gas pressure that satisfies the equation of state, $P \equiv(\gamma-1)\left(E-\rho \frac{v^{2}}{2}-\frac{B^{2}}{2}\right)$. The divergence-free constraint $\boldsymbol{\nabla} \cdot \boldsymbol{B}=0$ is satisfied by the flux constrained transport (CT) [7].

A second-order accurate (in space and time) total variation diminishing (TVD) method [27] is used for solving the ideal MHD equations. In this method, we first hold the magnetic field fixed and then update the fluid variables. Then we perform a reverse procedure to complete a one time step. The three dimensional problem is solved by splitting it into one-dimensional sub-problems using a Strang-type directional splitting [36]. Here we summarize the numerical method. The ideal MHD equations can be written in flux-conservative vector form by considering the advection along the $x$ direction as follows

$$
\frac{\partial \boldsymbol{u}}{\partial t}+\frac{\partial \boldsymbol{F}(u)}{\partial x}=0
$$

where the conserved fluid variables and the fluxes vectors are given by

$$
\boldsymbol{u}=\left(\begin{array}{c}
\rho \\
\rho v_{x} \\
\rho v_{y} \\
\rho v_{z} \\
E
\end{array}\right), \quad \boldsymbol{F}=\left(\begin{array}{c}
\rho v_{x} \\
\rho v_{x}^{2}+P^{*}-B_{x}^{2} \\
\rho v_{x} v_{y}-B_{x} B_{y} \\
\rho v_{x} v_{z}-B_{x} B_{z} \\
\left(E+P^{*}\right) v_{x}-B_{x} \mathbf{B} \cdot \mathbf{v}
\end{array}\right)
$$


Equation (5) is solved by Jin and Xin's relaxing TVD method [13]. The fluxes are obtained using a monotone upwind scheme for conservation laws (MUSCL) with a second-order TVD (van Leer limiter) correction. A second-order RungeKutta scheme is used to get the time integration. Let $L_{i}$ be the update operator of $\boldsymbol{u}^{t}$ to $\boldsymbol{u}^{t+\Delta t}$ by including the flux along $i$ direction in a time step $\Delta t$. Each $L_{i}$ includes three update operations in sequence, for example, $L_{x}$ includes the fluid update along $x$, the update of $B_{y}$ along $x$, and the update of $B_{z}$ along $x$. A forward sweep and a reverse sweep are defined as $\boldsymbol{u}^{t+\Delta t}=L_{z} L_{y} L_{x} \boldsymbol{u}^{t}$ and $\boldsymbol{u}^{t+2 \Delta t}=L_{x} L_{y} L_{z} \boldsymbol{u}^{t+\Delta t}$, respectively. Then the dimensional splitting of the relaxing TVD can be expressed as follows

$$
\begin{aligned}
& \boldsymbol{u}^{t_{2}}=\boldsymbol{u}^{t_{1}+2 \Delta t_{1}}=L_{x} L_{y} L_{z} L_{z} L_{y} L_{x} \boldsymbol{u}^{t_{1}} \\
& \boldsymbol{u}^{t_{3}}=\boldsymbol{u}^{t_{2}+2 \Delta t_{2}}=L_{z} L_{x} L_{y} L_{y} L_{x} L_{z} \boldsymbol{u}^{t_{2}} \\
& \boldsymbol{u}^{t_{4}}=\boldsymbol{u}^{t_{3}+2 \Delta t_{3}}=L_{y} L_{z} L_{x} L_{x} L_{z} L_{y} \boldsymbol{u}^{t_{3}}
\end{aligned}
$$

where $\Delta t_{1}, \Delta t_{2}$, and $\Delta t_{3}$ are sequential time steps after each double sweep. The operator $L_{i}$ contains the majority of the calculations in the numerical method. Since the updates in $L_{i}$ are first performed along the $x$ direction, then $y$, and then $z$, the numerical kernel only has to be implemented once and can be used for all directions by changing the targeting index.

\section{MHD simulations on a single GPU}

In this section, we briefly review our code $G P U-M H D$ for MHD simulations on a single GPU, for further information, please refer to [43]. GPU-MHD was designed to be run entirely on GPUs and it supports both single and double precision. Here we summarize our strategies for the design as follows:

- One or several CUDA kernels were designed and implemented for each step of the numerical scheme to exploit the parallelism of GPUs;

- Storage of the intermediate results (such as the "flux" and some interpolated values of each grid point), which will not be used in the next calculation step, are reused to reduce memory usage;

- In order to provide effective memory access, the eight components $\left(\rho, \rho v_{x}\right.$, $\left.\rho v_{y}, \rho v_{z}, E, B_{x}, B_{y}, B_{z}\right)$ of the MHD equations are stored in separate arrays and each component of a grid point is stored close to the same component of the neighboring grid points. In addition, only the necessary component of a calculation kernel will be accessed.

For memory arrangement of $G P U-M H D$, we store the data in one-dimensonal arrays and perform the parallel computation with one-dimensional threads due to the limited support of multidimensonal threads by CUDA. Figure 2 of [43] 
shows the data storage arrangement of GPU-MHD. The storage and threading method can be extended to solve multidimensional problems using an indexing technqiue shown in Equations (26) to (29) of [43]. The flow chart of GPU$M H D$ can be found in Figure 3 of [43], where you can see the calculations of sweeping operations $L_{x}, L_{y}$, and $L_{z}$ form the core part of the implementation. Each sweeping operation $L_{i}$ will update both the fluid variables and orthogonal magnetic fields along the $i$ dimension. In Figure 4 of [43], where you can see each block was implemented with one or several CUDA kernels. The algorithms for updating the fluid variables and magnetic components can be found in Algorithm 1 and Algorithm 2 of [43], respectively. As the only difference among $L_{x}, L_{y}$, and $L_{z}$ is the dimensional index, we just need to change the targeting index to get a generic sweeping operator $L_{i}$ work for a particular dimension by performing indexing operations in all $L_{i}$ kernels. Figure 5 of [43] shows the flux computation of GPU-MHD. Once the whole pipeline in Figure 3 of [43] is completed, the MHD simulation results will be stored in graphics memory (GRAM) and these results are readily to be visualized or read back to the CPU for other usage.

\section{Efficient GPU implementation on distributed multi-GPU sys- tems}

Numerical simulations using GPUs usually can achieve high efficiency, however, the limited size of the memory on a graphics card restricts the resolution of a MHD simulation that runs on a single GPU. According to the ideal MHD equations, there are eight physics quantities $\left(\rho, \rho v_{x}, \rho v_{y}, \rho v_{z}, E, B_{x}, B_{y}, B_{z}\right)$ for the 3D simulation. Extra memory for storing the intermediate results of the numerical method is required. In our experiment, the maximum resolution of a MHD simulation that runs on a NVIDIA Tesla M2050 GPU with 3GB memory is $225^{3}$. Therefore, distribute multi-GPU systems are needed for large-scale MHD simulations.

In a MHD simulation, the physics quantities of a grid point are updated by the calculation using one or several physics quantities of its adjacent grid points, and such calculation is called the stencil calculation. When performing simulations on clusters, the whole calculation domain is divided into many partitions and each partition is stored in a node. When calculating the elements on the boundary side of a partition, boundary elements from the adjacent partitions are needed as the inputs for the stencil calculation. Hence, a node needs a copy of the boundary elements of its adjacent partitions in every direction. The copy of the required elements of the adjacent partitions are called the halo (See Figure 1). The halo will be updated in every calculation step by copying the boundary data from the adjacent partitions. Therefore, data exchanges between GPUs and nodes are required, leading to the overhead in every step 


\subsection{Overlapping}

Overlapping is now a common technique used in GPU computing. Similar to the direct memory access (DMA) mechanism of the host system, NVIDIA GPUs with Compute Capability higher than 1.1 version contain dedicated DMA engine for data transfers over PCIe that work concurrently with CUDA kernel executions. The concept of overlapping for grid-based MHD simulations is to calculate the boundary elements first. Then, renew the halo by copying the updated boundary elements between every partition and calculating the interior grid points simultaneously (see Figure 2).

Overlapping causes some overheads because of breaking the computation into two steps. The processing time of a kernel is usually $T_{\text {Non-Overlapped }}<T_{\text {Overlapped }}$. Nevertheless, overlapping can hide the latency of I/O, resulting in a performance gain in total. The time expense can be calculated by the following equations with different conditions:

$$
T_{\text {Non-Overlapped }}<T_{\text {Overlapped }}=T_{\text {Boundary }}+T_{\text {Interior }}
$$

Without overlapping:

$$
T_{\text {Total }}=T_{\text {Non-Overlapped }}+T_{\text {DataExchange }}
$$


Fig. 2. The concept of overlapping: Time expense of Equation (12) (top); Time expense of Equation (13) (bottom).

Overlapping where $T_{\text {DataExchange }} \leq T_{\text {Interior }}$ :

$$
T_{\text {Total }}=T_{\text {Overlapped }}=T_{\text {Boundary }}+T_{\text {Interior }}
$$

Overlapping where $T_{\text {DataExchange }} \geq T_{\text {Interior }}$ :

$$
T_{\text {Total }}=T_{\text {Boundary }}+T_{\text {DataExchange }}
$$

It will be an ideal situation if the data exchange can be totally hidden by the calculation of the interior grid points (Equation (12)). This situation can happen in GPU applications on a single workstation with multiple GPUs. However, the time for data exchanges between nodes on a cluster is generally longer then the calculation time, and it is much longer for distributed multiGPU systems. The data of the simulation is stored in the dedicated memory on the graphics board, a GPU-CPU-GPU copy is required. That means the data have to be copied from the "device" (GPU) memory to the "host" (CPU) memory in a node (so-called "D2H copy"), and then transfer to another node, and copy from the "host" memory to the "device" memory (so-called "H2D copy") of the another. In addition, the amount of the exchanging data is large in MHD simulation. Therefore, the total time expense of GPU-MHD simulation on distributed multi-GPU systems is described by Equation (13).

In this case, the calculation of the interior is hidden by the data transfer. The larger $T_{\text {Interior }}$ compared to the $T_{\text {Boundary }}$, the better performance will 

size of the halos (= 4 in the TVD MHD method). However, the evolution of the physics quantities of the numerical simulation in a $d t$ requires many calculation steps. And the stencil calculation of each step needs one or more adjacent grid points invoked. In our implementation, we need four boundary elements in each direction to be updated before the overlapping process of the data transfer and the calculation of interior. To update four fluid vectors $\boldsymbol{u}$ of the MHD equations, $4+1=5$ fluxes $\boldsymbol{F}$ are needed to be calculated first. To get five fluxes, more intermediate results calculated by the boundary elements are required. Even we only need four updated $u$. The number of the boundary elements of the intermediate results of each step is an increment number counting from the last process block to the first process block of Figure 2 . For both the fluid and the magnetic field parts of every $L_{i}(i=x, y, z)$, and the concept is illustrated in Figure 3. The total number of computed elements is the same as non-overlapped approach. But the increasing number of the precomputed boundary elements affects the performance gain of the overlapping technique.

$L_{x}, L_{y}$ and $L_{z}$ of $G P U-M H D$ contains the same calculation kernels with different indexing operations (see Figure 5 of [43]). To extend the computation of $L_{i}$ with overlapping, we only need new index operations for the traversal of the boundary elements and the interior elements. The calculation parts are still the same for both boundary and interior calculations of $L_{x}, L_{y}$, and $L_{z}$, as shown in Figure 4. Overlapping somehow enhances the efficiency, but due to the high workload of data exchanges, a better method is still needed to speedup the data exchanges.

\subsection{GPU Direct-MPI Hybrid Approach}

Distributed multi-GPU systems usually have more than one GPU installed on the PCIe interfaces in each node. Before GPU Direct 2.0 is available, data exchanges between two GPUs have to proceed a GPU-CPU-GPU memory copy. This indirect way dramatically reduces the performance of distributed 

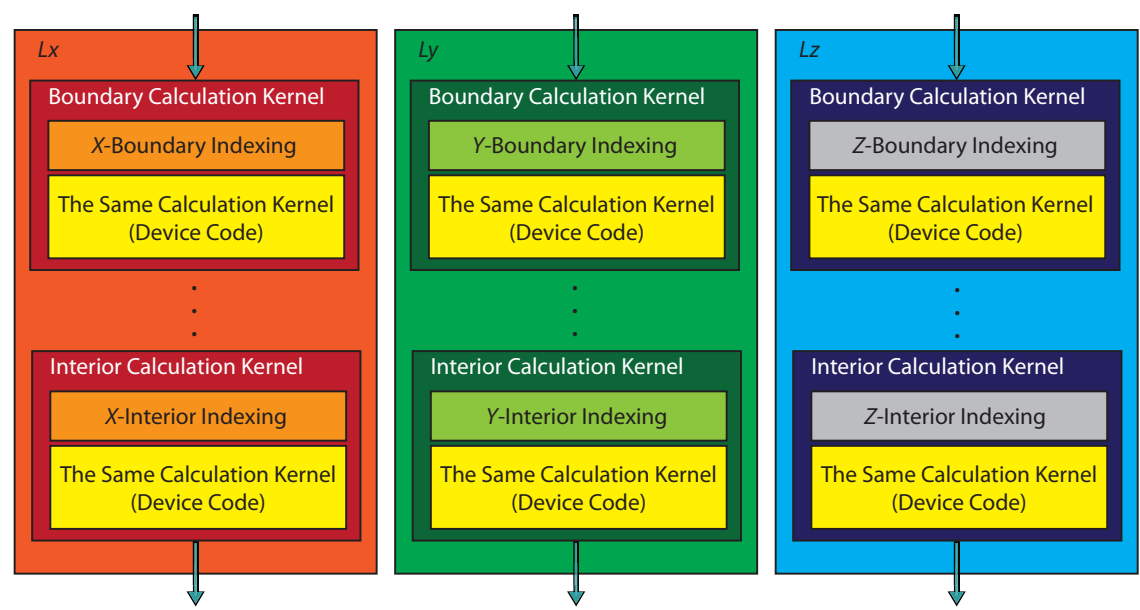

Fig. 4. Computation kernels in $M G P U-T V D$ for overlapping.

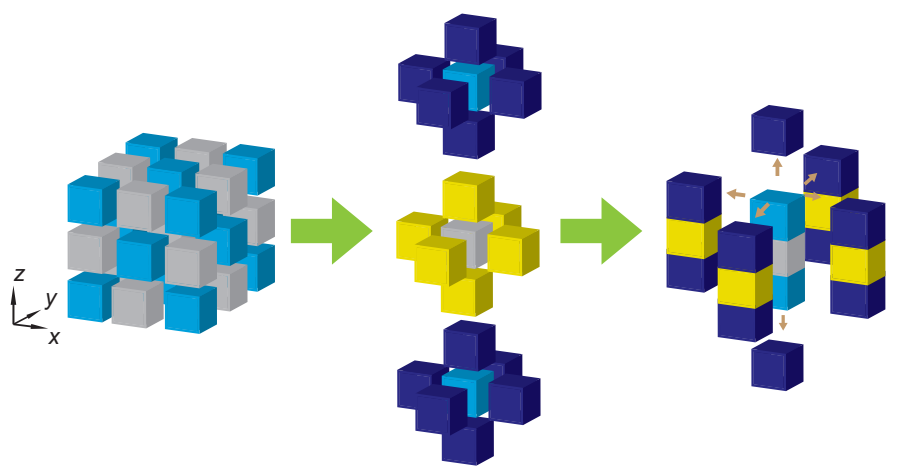

Fig. 5. Multiple partitions in the $z$-dimension can be handled by multiple GPUs in one single process.

multi-GPU systems. The overhead is large and cannot be hidden by overlapping technique. In order to enhance the performance of large-scale MHD simulations on distributed multi-GPU systems, we hereby propose a GPU Direct-MPI hybrid approach. In this approach, MPI is only used for the data exchange between computer nodes where GPU Direct 2.0 is applied for the internal data exchange between GPUs within a node. It is different from the common used MPI-only approach which launches the same number of processes as the number of GPUs, we only need to launch one process in each node to drive all the GPUs in a node. Therefore, the computational domain is divided by the number of node rather than the total number of GPUs of the system. As a result, only two MPI data transfers on each direction are needed per node, as showed in Figure 5. Our approach reduces the MPI traffic and achieves higher efficiency in data exchanges, resulting in total efficiency enhancement since the data exchange is the bottleneck of large-scale MHD simulations on distributed multi-GPU systems. More details of the strategies used in our approach: (1) Using GPU Direct for fast internal data exchange between GPUs (2) Using CUDA kernel to speedup the fragmented data exchange, will be explained in the following subsections. 


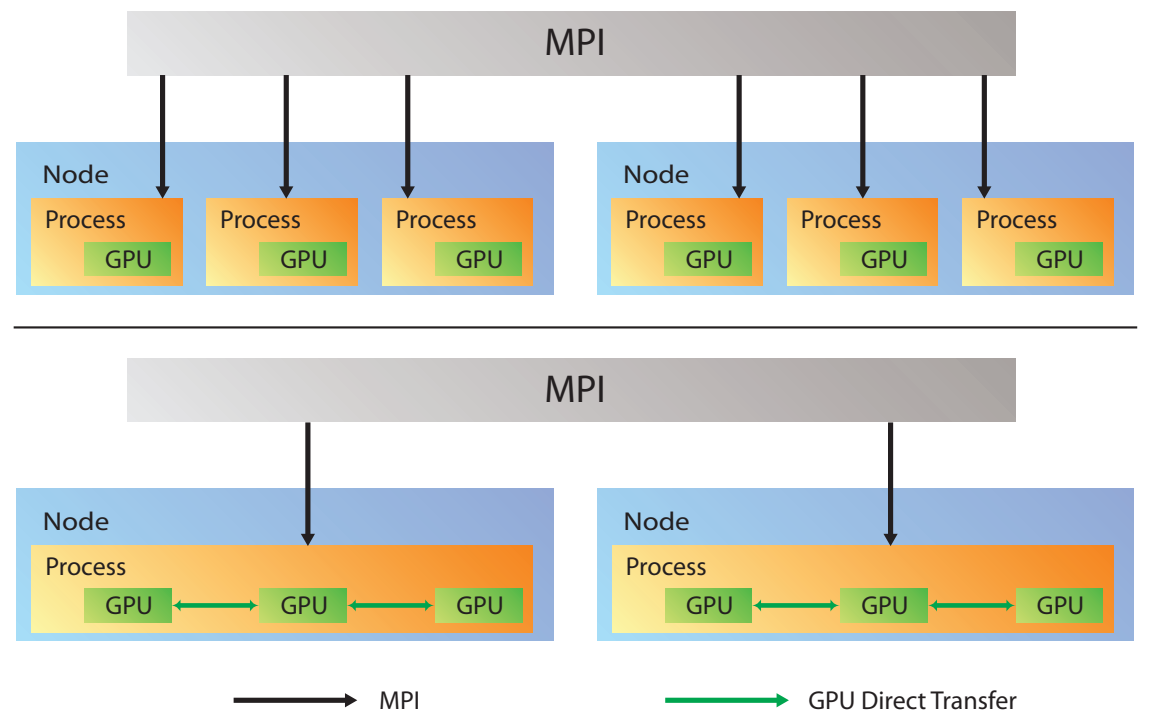

Fig. 6. Comparison between using MPI-only (top) and GPU Direct 2.0 (bottom) for inter-node data exchanges.

\subsubsection{GPU peer-to-peer data transfers}

GPU Direct 2.0 is a feature of Fermi or newer generation GPUs and it is available for CUDA 4.0 or later version. It provides peer-to-peer data communications between two GPUs in the same node. By using GPU Direct 2.0, data transfers between two GPUs become straightforward and much efficient. On the other hand, a single process can handle all the GPUs in a node in a easier way by using CUDA runtime APIs. Figure 6 shows the different between two approaches of using MPI-only and GPU Direct 2.0 for data communications between GPUs.

Because multiple GPUs within a node are handled by one single process, the decomposition of the computational domain becomes a two-layer process. At the first layer, the whole computational domain is decomposed by the number of computation node of the system, assigned to every node and communicated using MPI. At the second layer, a partition domain in a node is decomposed in one dimension ( $z$-dimension in our case) and assigned to every GPU. For the data exchange in the $z$-direction, only the first GPU and last GPU have to exchange the data to the adjacent node via MPI (see Figure 5). The data exchanges in $y$ and $x$ directions can be done by packing the data to a single buffer and transferring the packed data to another nodes via MPI. When a node receives the packed data, it unpacks the data and then distributes them to every GPU in that node. More detail about the data exchange in $y$ and $x$ directions will be explain in the following subsection. The data exchange between GPUs in $z$ direction in the same node is done via GPU peer-to-peer data transfer. The cudaMemcpyAsync CUDA API is used to exchange the data inside a node instead of MPI_isend and MPI_irecv (or MPI_sendrecv). This approach not only increases the efficiency of the data exchange between the 
GPUs inside a node, but also reduces the number of MPI communications. Asynchronous copy (non-blocking copy with synchronization) is used to let the GPUs transfer the data concurrently. Furthermore, internal data exchanges using GPU Direct 2.0 transferring data between GPUs via PCIe interface directly. Thus, it can be run simultaneously with MPI transfers. Experimental results shows the great benefit of our approach compared to common MPI-only approach.

\subsubsection{Moving the fragmented data via CUDA kernel}

To run large-scale MHD simulations using many nodes on a distribute multiGPU system, it is necessary to decompose the whole computational domain into a certain number of partitions and assign each partition to a node for computation. For 3D MHD simulations, in theory, the best way is to use 3D decomposition since each partition will have smallest surface area. In other words, each partition will have less halo elements which means less memory usage for the halo and less data to be transferred. Thus, it shortens the data exchange time. Besides, since the memory usage of the halo is reduced, the saved memory can be use for the non-halo data. More grid points can be calculated in each node. Table 1 shows an example of the halos of a 3D MHD simulation with the resolution $=1080^{3}$ using different decomposition methods. There are eight quantities and four halos in each direction. The calculation of the memory usage of a node can be simply obtained by the following equations:

$$
\begin{aligned}
& \text { PartitionSize }_{\text {withoutHalo }}=\quad \text { PartitionSize }_{x} \\
& \times \text { PartitionSize }_{y} \\
& \times \text { PartitionSize } \\
& \text { PartitionSize }_{\text {withHalo }}=\quad\left(\text { PartitionSize }_{x}+2 \times \text { Halo }\right) \\
& \times\left(\text { PartitionSize }_{y}+2 \times \text { Halo }\right) \\
& \times\left(\text { PartitionSize }_{z}+2 \times \text { Halo }\right) \\
& =\quad\left(\text { PartitionSize }_{x}+8\right) \\
& \times\left(\text { PartitionSize }_{y}+8\right) \\
& \times\left(\text { PartitionSize }_{z}+8\right) \\
& \text { NumberOfHalo }=\quad \text { NumberOfQuantities } \\
& \times(\text { PartitionSize } \text { withHalo } \\
& \text {-PartitionSize withoutHalo }) \\
& =\quad 8 \times\left(\text { PartitionSize }_{\text {withHalo }}\right. \\
& \text {-PartitionSize withoutHalo }) \\
& \text { MemoryUsage }=(\text { NumberOfHalo }) \times(\text { BytesOfVariable }) \\
& =(\text { NumberOf Halo }) \times \frac{8}{1024 \times 1024 \times 1024}
\end{aligned}
$$


Fig. 7. Data addresses on the memory: Boundary data in the $x$-boundary is fragmented and shows poor performance in memory copy.

Table 1

The number of HALO elements and its memory usage (double precision) in different decomposition methods of a $1080^{3}$ MHD simulation.

\begin{tabular}{|c|c|c|}
\hline Decomposition & Number of the halo per node & Memory usage (GBytes) \\
\hline $1 \times 1 \times 216$ & 76453376 & 0.56962204 \\
$1 \times 12 \times 18$ & 11347456 & 0.084545135 \\
$6 \times 6 \times 6$ & 6501376 & 0.048439026 \\
\hline
\end{tabular}

where PartitionSize is the size of a partition domain. For example, PartitionSize of the $1080^{3}$ domain with $6 \times 6 \times 6$ decomposition is:

$\left(\right.$ PartitionSize $_{x}$, PartitionSize $_{y}$, PartitionSize $\left._{z}\right)=(1080 / 6,1080 / 6,1080 / 6)=$ $(180,180,180)$.

Unfortunately, the I/O of the fragmented data of the device memory is extremely low performance (see Figure 7). In our experience, it spent dozens or even hundreds of times for copying the halo data in $x$-direction compared to $z$-direction. However, we found that moving the data via a CUDA kernel (simply launch a " $a=b$ " CUDA kernel) preforms a fast data copy. This improves the efficiency of the data transfer in the 3D decomposition. As a result, 3D decomposition becomes useful and is used in our large-scale MHD simulation implementation. Moreover, it shows higher efficiency than the common used 2D decomposition approach.

The procedures of the data exchange in $x$ and $y$-directions are showed as follows and in Figure 8.

(1) For each GPU, copy the data beside the halo to a continue/linear memory space of the same GPU.

(2) All GPUs copy the data to one single host buffer via asynchronous copy.

(3) Copy host memory of the combined data to adjacent node via MPI.

(4) Copy the data from the host buffer to a continue/linear memory space of 
Fig. 8. Data exchange in $x$ and $y$ directions. Data copy between device memory of each GPU and host memory are concurrent.

each GPU via asynchronous copy.

(5) For each GPU, copy the data to the halo from the continue/linear memory space of the same GPU.

Copying the fragmented data directly from device to host is very slow. A buffer is used to align the data before the D2H and H2D copy. GPU has high bandwidth in device-to-device (D2D) copy. Copying data to a linear buffer and then proceeding the $\mathrm{D} 2 \mathrm{H}$ or $\mathrm{H} 2 \mathrm{D}$ copy shows better performance than a $\mathrm{D} 2 \mathrm{H} / \mathrm{H} 2 \mathrm{D}$ copy directly with the discontinued memory space of the device memory.

In Steps 1 and 5, D2D copy is done within each GPU concurrently. For the D2D copy in each GPU, CUDA asynchronous copy is used in $y$-direction where CUDA kernel is used in $x$-direction. As we mentioned, data copy of the fragmented data in $x$-direction is very slow but it is fast if we use CUDA kernel. In our experimental results, the average transfer rates of a whole process of the data copy including all the five steps we listed above are $0.153 \mathrm{~GB} / \mathrm{s}$ in $y$-direction and $0.174 \mathrm{~GB} / \mathrm{s}$ in $x$-direction. The data exchange in $x$-direction can even provide faster speed compared to $y$-direction in a breakdown measurement. However, since the CUDA kernel is launched for the data exchange in $x$-direction, overlapping technique cannot be applied for the data exchange in $x$-direction. So we use data copy with overlapping for $y$-direction and $z$ direction. For $x$-direction, non-overlapped and CUDA kernel data copy are used.

In Step 2, each GPU copies the data to the buffer to a single host memory with different starting points of the address. The packed data from all the GPU will be transferred to another node via one MPI transfer process. After that, the packed data will be distributed to each GPU on the other node using a D2H copy. CUDA asynchronous copy are used in Steps 2 and 4, allowing multiple GPUs copy data to/from the host buffer simultaneously. 
Using CUDA kernels for fragmented data exchanges instead of cudaMemcpy is simply but the benefit is huge. In common MPI-only approach on distributed multi-GPU systems, 3D decomposition is always much slower than 2D decomposition due to the low efficient fragmented data exchange. The breakthrough of making 3D decomposition available on distributed multi-GPU systems, minimizing the number of halos in large-scale MHD simulations, resulting in a performance gain in both data exchanges and calculations.

\section{$5 \quad$ Numerical tests}

In this section, we present several $2 \mathrm{D}$ and $3 \mathrm{D}$ numerical tests for validating our implementation. All tests were performed in double precision on the TSUBAME 2.0 supercomputer at the Tokyo Institute of Technology.

\subsection{MHD rotor problem}

The first test is the 2D MHD rotor problem taken from [38]. It initiates a high density rotating disk with radius $r_{0}=0.1$ of fluid measured from the center point $(x, y)=(0.5,0.5)$. The ambient fluid outside of the spherical region of $r_{1}=0.115$ ) has low density and $v_{x}=v_{y}=0$, and the fluid between the high density disk fluid and ambient fluid $\left(r_{1}>r>r_{0}\right.$, where $\left.r=\sqrt{(x-0.5)^{2}+(y-0.5)^{2}}\right)$ has linear density and angular speed profile with $\rho=1+9 f, v_{x}=-f v_{0}(y-0.5) / r$ and $v_{y}=f v_{0}(x-0.5) / r$ where $f=\left(r_{1}-r\right) /\left(r_{1}-r_{0}\right)$. The initial condition is listed as follows

$$
\begin{aligned}
& r<r_{0} \\
& \left\{\begin{array}{l}
v_{x} \\
v_{y} \\
v_{z}
\end{array}\right\}=\left\{\begin{array}{c}
-v_{0}(y-0.5) / r_{0} \\
v_{0}(x-0.5) / r_{0} \\
0
\end{array}\right\} \\
& r_{0}<r<r_{1} \\
& \left\{\begin{array}{l}
v_{x} \\
v_{y} \\
v_{z}
\end{array}\right\}=\left\{\begin{array}{c}
-f v_{0}(y-0.5) / r \\
f v_{0}(x-0.5) / r \\
0
\end{array}\right\} \\
& \left\{\begin{array}{l}
v_{x} \\
v_{y} \\
v_{z}
\end{array}\right\}=\left\{\begin{array}{l}
0 \\
0 \\
0
\end{array}\right\}
\end{aligned}
$$




$$
\rho=\left\{\begin{array}{cc}
10 & r<r_{0} \\
1+9 f & r_{0}<r<r_{1} \\
1 & r>r_{1}
\end{array}\right.
$$

spherical region center $=(0.5,0.5)$

$$
r_{0}=0.1, r_{1}=0.115
$$

$$
f=\left(r_{1}-r\right) /\left(r_{1}-r_{0}\right),(0 \leq x \leq 1),(0 \leq y \leq 1)
$$

A set of initial value of $v_{0}, p, B_{x}$ and $\gamma$ provided in [38] was tested

$$
v_{0}=1, \quad p=0.5, \quad \gamma=5 / 3, \quad\left\{\begin{array}{c}
B_{x} \\
B_{y} \\
B_{z}
\end{array}\right\}=\left\{\begin{array}{c}
2.5 / \sqrt{4 \pi} \\
0 \\
0
\end{array}\right\}
$$

Figure 9 presents the images of the density computed with $600 \times 600$ grid points. The results are in excellent agreement with those presented in [38].

\subsection{Blast wave problem}

The second test is the 2D MHD blast wave problem. The MHD spherical blast wave problem of Zachary et al. [46] is initiated by an over pressured region in the center of the domain. The result is a strong outward moving spherical shock with rarified fluid inside the sphere. We followed the test suite [31] of Athena [35]. The initial condition for 2D MHD blast wave problem is listed as follows [31]

$$
\begin{gathered}
\left\{\begin{array}{c}
v_{x} \\
v_{y} \\
v_{z}
\end{array}\right\}=\left\{\begin{array}{l}
0 \\
0 \\
0
\end{array}\right\} \\
\left\{\begin{array}{c}
B_{x} \\
B_{y} \\
B_{z}
\end{array}\right\}=\left\{\begin{array}{c}
1 / \sqrt{2} \\
1 / \sqrt{2} \\
0
\end{array}\right\}
\end{gathered}
$$


ig. 9. Results of the density of the 2D MHD rotor problem computed with $600 \times$ 600 grid points at $t=0.01 \mathrm{~s}$ (top-left), 0.09s (top-right), $0.17 \mathrm{~s}$ (bottom-left), and $0.25 \mathrm{~s}$ (bottom-right).

$$
\begin{aligned}
& \rho=1, \quad \gamma=5 / 3, \quad p=\left\{\begin{array}{cc}
10 & \text { inside the spherical region } \\
0.1 & \text { outside the spherical region }
\end{array}\right. \\
& \text { spherical region center }=(0.5,0.5), r=0.1 \\
& (0 \leq x \leq 1), \quad(0 \leq y \leq 1)
\end{aligned}
$$

In Figure 10, we present images of the density computed with $600 \times 600$ grid points. The results are in excellent agreement with those presented in [31].

The third test is the 3D MHD spherical blast wave problem. The velocity, density $\rho$, pressure $p$, and $\gamma$ are the same as those in 2D case. The different 
Fig. 10. Results of the density of the 2D blast wave problem computed with 600 $\times 600$ grid points at $t=0.01 \mathrm{~s}$ (top-left), $0.16 \mathrm{~s}$ (top-right), $0.29 \mathrm{~s}$ (bottom-left), and $0.38 \mathrm{~s}$ (bottom-right).

part of the initial condition is listed as follows [31]

$$
\left\{\begin{array}{c}
B_{x} \\
B_{y} \\
B_{z}
\end{array}\right\}=\left\{\begin{array}{c}
1 / \sqrt{3} \\
1 / \sqrt{3} \\
1 / \sqrt{3}
\end{array}\right\}
$$

$$
\begin{aligned}
& p=\left\{\begin{array}{l}
10 \text { inside the spherical region } \\
0.1 \text { outside the spherical region }
\end{array}\right. \\
& \rho=1, \quad \gamma=5 / 3
\end{aligned}
$$

spherical region center $=(0.5,0.5,0.5), r=0.1$

$$
(0 \leq x \leq 1),(0 \leq y \leq 1),(0 \leq z \leq 1)
$$


Figure 11 shows the results of the density computed with $300 \times 300 \times 300$ grid points. Due to the scarcity of published 3D test results, we do not make direct contact with results presented in the literature here.

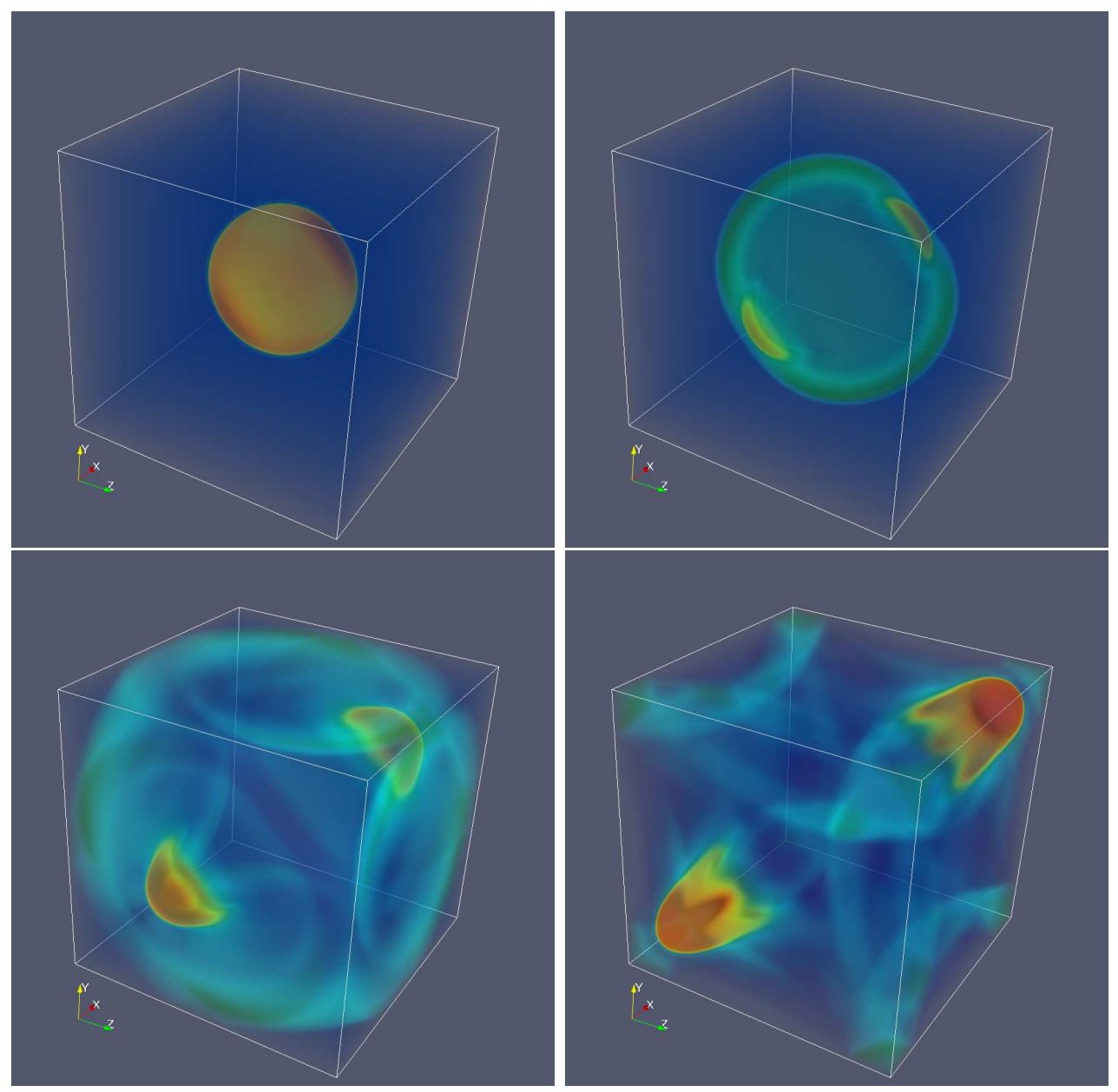

Fig. 11. Results of the density of the 3D blast wave problem computed with $300 \times$ $300 \times 300$ grid points at $t=0.07 \mathrm{~s}$ (top-left), $0.18 \mathrm{~s}$ (top-right), $0.41 \mathrm{~s}$ (bottom-left), and $0.62 \mathrm{~s}$ (bottom-right).

\subsection{Orszag-Tang problem}

The fourth test is the 2D Orszag-Tang vortex problem [24], which is used to study incompressible MHD turbulence. In our test, the boundary conditions are periodic everywhere. The density $\rho$, pressure $p$, initial velocities $\left(v_{x}, v_{y}, v_{z}\right)$, 
and magnetic field $\left(B_{x}, B_{y}, B_{z}\right)$ are given by

$$
\begin{gathered}
\left\{\begin{array}{l}
v_{x} \\
v_{y} \\
v_{z}
\end{array}\right\}=\left\{\begin{array}{c}
-\sin (2 \pi y) \\
\sin (2 \pi x) \\
0
\end{array}\right\} \\
\left\{\begin{array}{c}
B_{x} \\
B_{y} \\
B_{z}
\end{array}\right\}=\left\{\begin{array}{c}
-B_{0} \sin (2 \pi y) \\
B_{0} \sin (4 \pi x) \\
0
\end{array}\right\} \text { where } B_{0}=1 / \sqrt{4 \pi} \\
\rho=25 /(36 \pi), \quad p=5 /(12 \pi), \quad \gamma=5 / 3, \quad(0 \leq x \leq 1), \quad(0 \leq y \leq 1)
\end{gathered}
$$

The 2D Orszag-Tang vortex test was performed in a 2D periodic box with 600 $\times 600$ grid points. The results of the density are shown in Figure 12, where the complex pattern of interacting waves is perfectly recovered. The results agree well with those in Lee et al. [16].

The fifth test is the 3D Orszag-Tang vortex obtained from [19], which is used to study the creation and evolution of small-scale structures in 3D MHD flows. In our test, the boundary conditions are periodic everywhere. The density $\rho$, pressure $p$, and $\gamma$ are the same as those in $2 \mathrm{D}$ case. Initial velocities $\left(v_{x}, v_{y}, v_{z}\right)$, and magnetic field $\left(B_{x}, B_{y}, B_{z}\right)$ are given by

$$
\begin{gathered}
\left\{\begin{array}{l}
v_{x} \\
v_{y} \\
v_{z}
\end{array}\right\}=\left\{\begin{array}{c}
-2 \sin (2 \pi y) \\
2 \sin (2 \pi x) \\
0
\end{array}\right\} \\
\left\{\begin{array}{c}
B_{x} \\
B_{y} \\
B_{z}
\end{array}\right\}=\left\{\begin{array}{c}
B_{0}[2 \sin (4 \pi y)+\sin (2 \pi z)] \\
B_{0}[2 \sin (2 \pi x)+\sin (2 \pi z)] \\
B_{0}[\sin (2 \pi x)+\sin (2 \pi y)]
\end{array}\right\} \text { where } B_{0}=1 / \sqrt{4 \pi} . \\
(0 \leq x \leq 1),(0 \leq y \leq 1),(0 \leq z \leq 1)
\end{gathered}
$$

The 3D Orszag-Tang vortex test was performed in a 3D periodic box with 300 $\times 300 \times 300$ grid points. The results of the density are shown in Figure 13, where the complex pattern of interacting waves is perfectly recovered. 

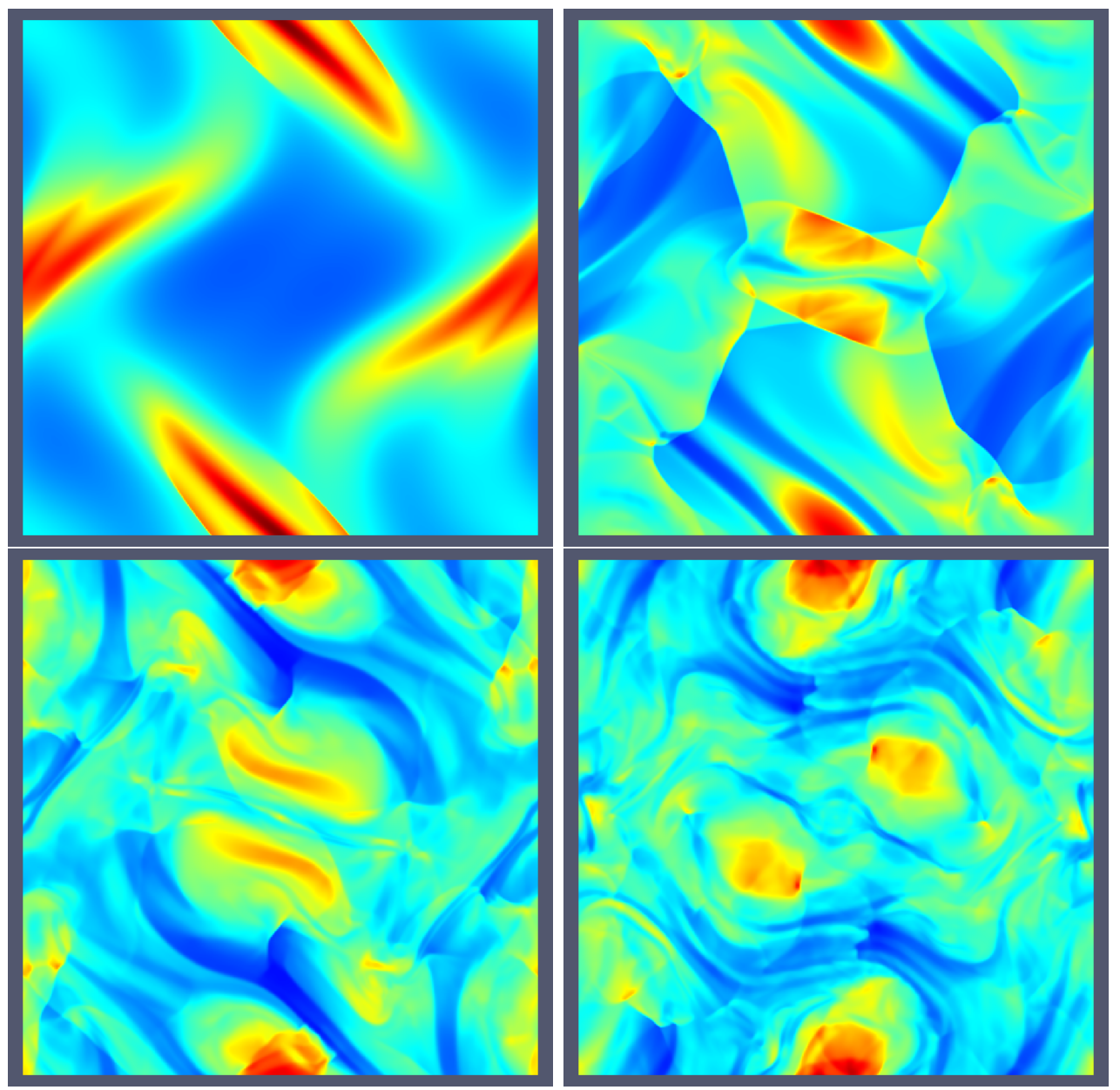

Fig. 12. Results of the density of the 2D Orszag-Tang vortex problem computed with $600 \times 600$ grid points at $t=0.15 \mathrm{~s}$ (top-left), 0.50s (top-right), $0.83 \mathrm{~s}$ (bottom-left), and $1.19 \mathrm{~s}$ (bottom-right).

\section{Performance measurements}

The performance measurements of our code - MGPU-MHD are carried out in this section. All the tests were performed in double precision on the TSUBAME 2.0 supercomputer $[6,11]$ at the Tokyo Institute of Technology using 216 NVIDIA Tesla M2050 GPUs (72 nodes). Before we analyze the performance, we briefly explain the configuration of a calculation node of the TSUBAME 2.0 shown in Figure 14. Each calculation node has three NVIDIA Tesla M2050 (Fermi architecture) GPUs installed and each GPU contains 3GB device memory. In fact, data transfers between GPU0 and GPU1 or GPU2 cannot be done via peer-to-peer transfer since there are two IOHs between them. CUDA will automatically switch to GPU-CPU-GPU data copy without the requirement of allocating extra host memory. The transfer rate between GPU0 and GPU1 or GPU2 is from 2.88 to $3.56 \mathrm{~GB} / \mathrm{s}$ and it is $4.9 \mathrm{~GB} / \mathrm{s}$ using peer-to-peer transfer between GPU1 and GPU2 on a calculation node of the TSUBAME 

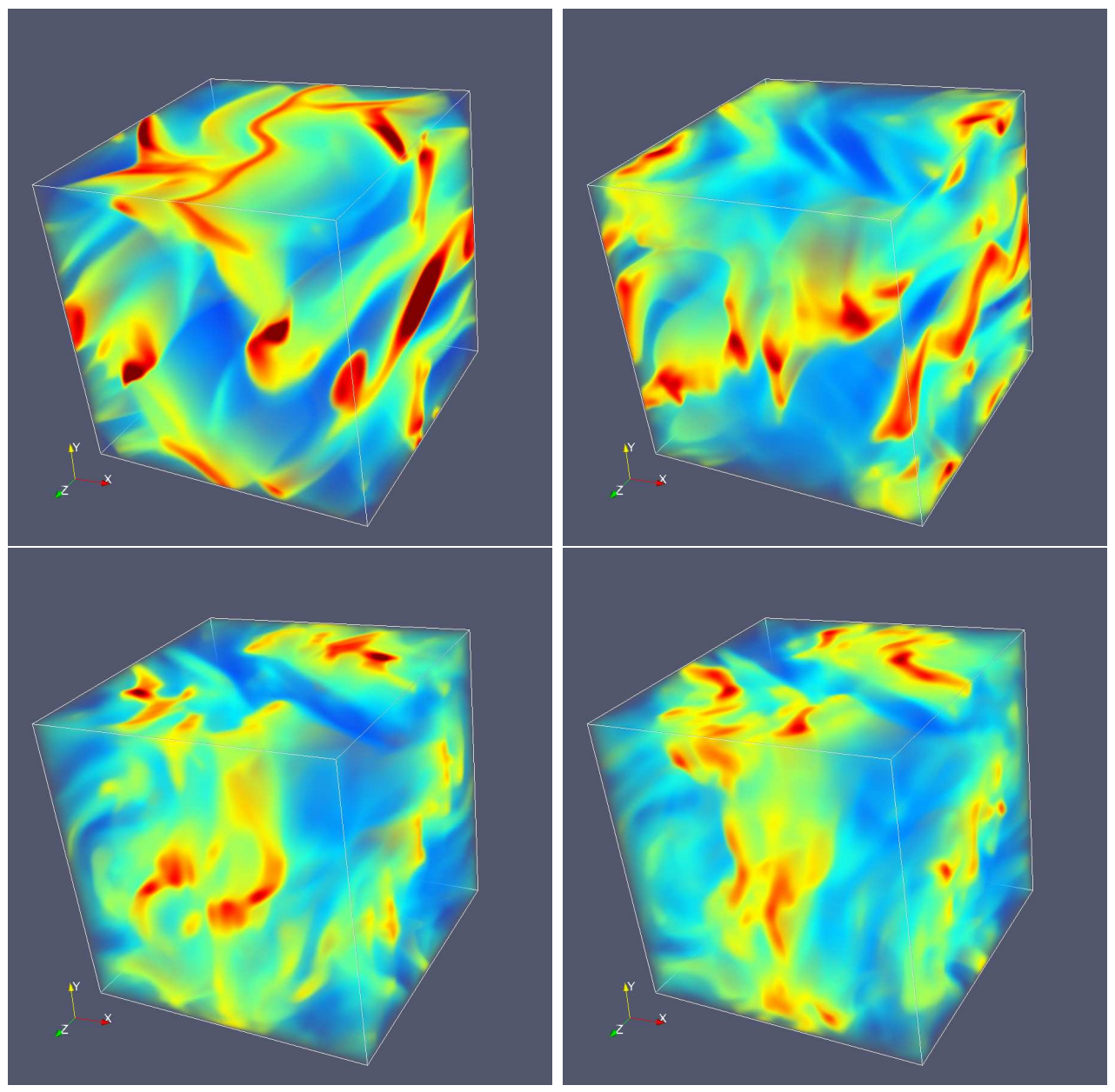

Fig. 13. Results of the density of the 3D Orszag-Tang vortex problem computed with $300 \times 300 \times 300$ grid points at $t=0.24 \mathrm{~s}$ (top-left), $0.35 \mathrm{~s}$ (top-right), $0.52 \mathrm{~s}$ (bottom-left), and $0.57 \mathrm{~s}$ (bottom-right).

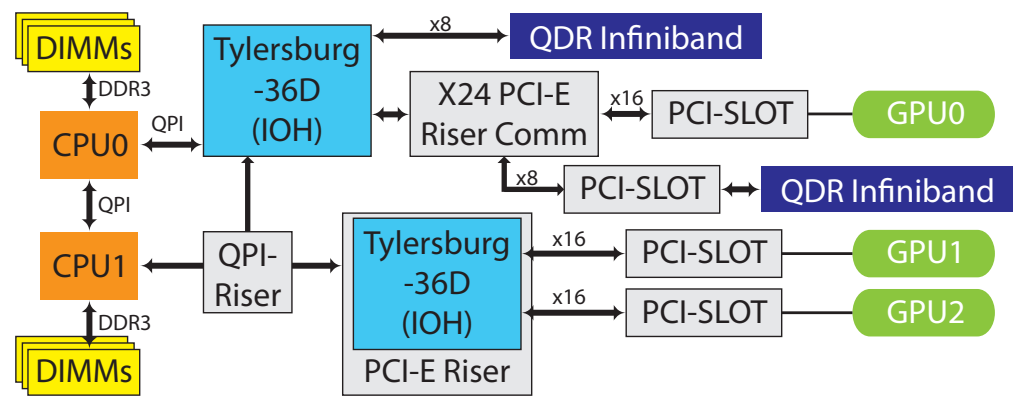

Fig. 14. The configuration of a calculation node of the TSUBAME 2.0 supercomputer [11].

\section{0 .}

Performance measurements are shown in Table 2 and Figure 15. The resolution of the whole computational domain was $864^{3}$ in all tests. By using CUDA kernels to move the halo data along the $x$-direction, simulations using the 3D 
Table 2

The comparison of different decomposition methods with respect to the elapse time (ms/step).

\begin{tabular}{|c|c|c|c|}
\hline Decomposition & MPI & GPU Direct & Speedup \\
\hline $6 \times 6 \times 6$ & 937.13 & 776.59 & 1.2 \\
$1 \times 18 \times 12$ & 1362.00 & 1092.80 & 1.25 \\
$1 \times 12 \times 18$ & 1299.46 & 974.05 & 1.33 \\
$1 \times 1 \times 216$ & 4708.47 & 3197.14 & 1.47 \\
\hline
\end{tabular}

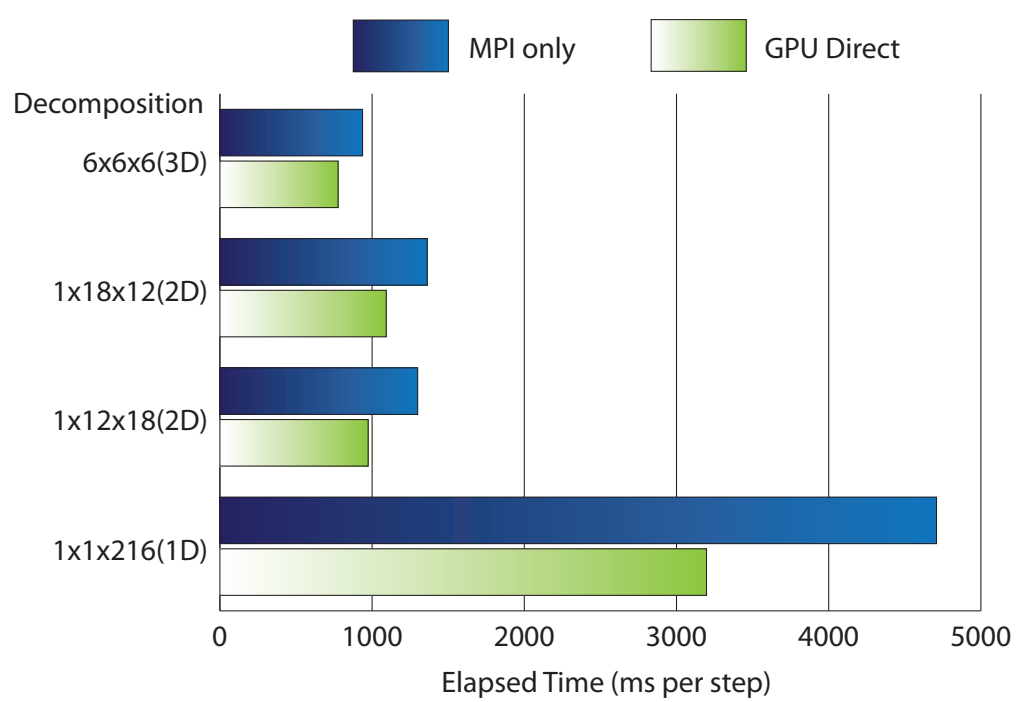

Fig. 15. The comparison of different decomposition methods with respect to the elapse time (ms/step) (Resolution: $864^{3}$ ).

decomposition achieved about $1.39 \times$ faster then those using $2 \mathrm{D}$ decomposition MPI-only method. Furthermore, we obtained about $1.2 \times$ speedup more $(1.67 \times$ in total) by using GPU Direct 2.0. Having the fastest elapse time, simulations using 3D decomposition with GPU Direct 2.0 showed enhancements in performance, supporting the benefits of our proposed method. In addition, $3 \mathrm{D}$ decomposition reduced the memory usage of the halo data, thus each GPU can calculate more data. In our tests using 216 GPUs, the maximum resolution of the whole computational domain that could be processed was $1200^{3}$ for $3 \mathrm{D}$ decomposition and performed 2 TFLOPS while 1D decomposition and 2D decomposition could only performed 0.42 TFLOPS and 1.4 TFLOPS, respectively. Without using our GPU Direct-MPI hybrid approach, the common 2D decomposition MPI-only implementation only got 1 TFLOPS. In conclusion, we got $2 \times$ the performance with our $3 \mathrm{D}$ decomposition method using GPU Direct-MPI hybrid approach.

Data exchange is a bottleneck preventing the improvement of the performance of numerical simulations on distributed multi-GPU systems. To further analyze our data transfer techniques for performance enhancements, tests and 
analysis of the data transfer of our GPU Direct-MPI hybrid approach using 216 GPUs were done. For breakdown measurements, we got the data exchange rate per node as $0.174 \mathrm{~GB} / \mathrm{s}, 0.153 \mathrm{~GB} / \mathrm{s}$, and $0.462 \mathrm{~GB} / \mathrm{s}$ along the $x-, y^{-}$, and $z$-directions, respectively. For the data exchanges along the $z$-direction, only the GPU0 or GPU3 is invoked in the data exchanges between nodes. The amount of data transferred via MPI is about $\frac{1}{3}$ of that of the total data exchange in the $x$ or $y$ direction. Data exchanges between GPUs in the same node are performed using the non-blocking and concurrent peer-to-peer transfers. In addition, data exchanges in different directions contain different overheads:

(1) In the $x$-direction - Fragmented data copy to linear memory via CUDA kernels.

(2) In the $y$-direction - Fragmented data copy to linear memory via CUDA memory copy.

(3) In the z-direction - Synchronization of the non-blocking memory between GPUs.

As a result, the data transfer rate of the data exchange along the $z$-direction is about $3 \times$ of that of the data exchange in the other two directions. Applying overlapping technique results in $11 \%$ performance gain. As we mentioned in Section 4.2.2, data exchanges along the $x$-direction block the overlapping. Overlapping is only applied to the $y$ - and $z$-directions. On the other hand, the number of the pre-computed boundary elements increases with respect to the number of the calculation steps (see Figure 3 in Section 4.1). $T_{\text {Boundary }}$ increases when $T_{\text {Interior }}$ decreases. Therefore, less overhead $\left(T_{\text {Interior }}\right.$ in this case) can be hidden by the overlapping. We consider that it restricts the performance enhancement of applying overlapping technique to 3D MHD simulations.

Figures 16 and 17 show the results of the data transfer efficiency of the whole data exchange process without the calculation part of our $M G P U-M H D$ code. It can be realized that we got a great benefit of the data exchange along the $z$-direction. This benefit increased if we have more partitions along the $z$-direction. The reason is that GPU Direct 2.0 was invoked for in-node GPU data exchanges. The larger number of $z$-decomposition the higher speedup we got, compared to the MPI-only approach. Besides, the $1 \times 72 \times 3$ decomposition (it is almost a 1D decomposition along the $y$-dimension) showed only a little bit slower because of the overhead of the data exchange along the $y$-direction. For the data exchanges along the $y$-direction, both MPI only and GPU Direct approaches copied the data into a linear buffer in the GPU. The overhead was caused by the synchronization of the non-blocking copies. The same overhead also existed in the data exchanges along the $x$-direction. According to these results, it showed evidently that this overhead was small and acceptable.

The above performance measurements show that our GPU Direct-MPI hybrid 
Fig. 16. The comparison of different decomposition methods with respect to the data transfer rate using 216 GPUs.

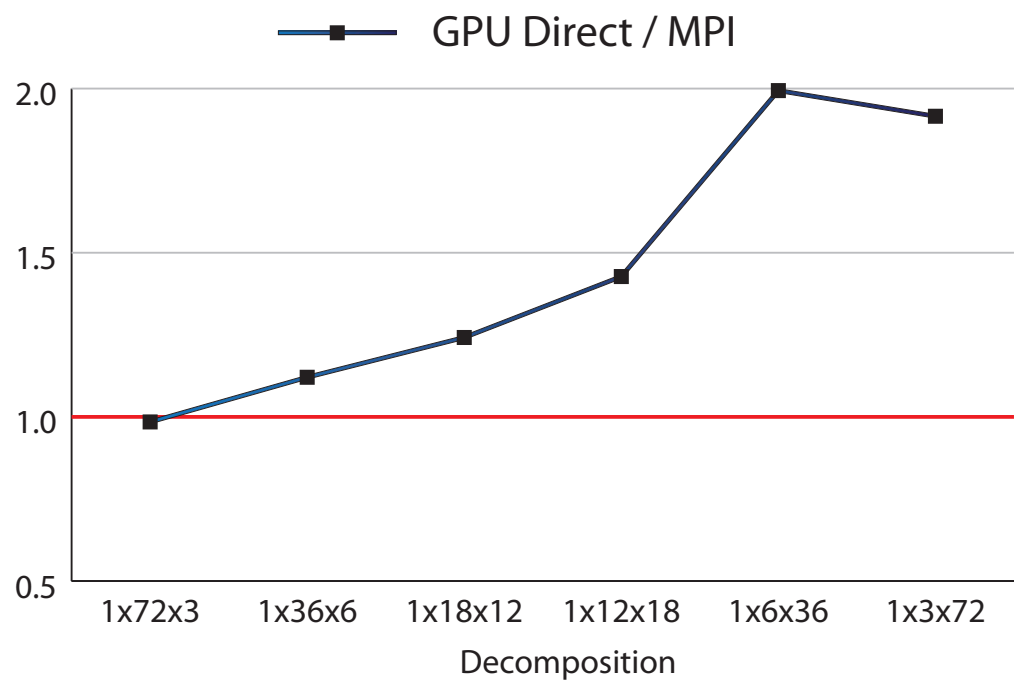

Fig. 17. The ratio of the data transfer rate between MPI-only approach and GPU Direct approach using 216 GPUs ( $\left.\frac{\text { GPUDirect }}{M P I}\right)$.

approach enhances the performance in many aspects, achieving great speedups of large-scale MHD simulations on distributed multi-GPU systems.

\section{Conclusion and future work}

Performing large-scale MHD simulations on distributed mult-GPU systems provide us an opportunity to obtain the simulation results more efficient, however, the overhead caused by the data transfer bottlenecks the simulation performance. Overcoming this problem will fully exploit the massive computational power of GPUs. In this paper we propose a novel GPU Direct-MPI hy- 
brid approach to address this problem. Our approach featuring two strategies: (1) We use GPU Direct 2.0 for peer-to-peer GPU data transfers to speedup the data exchange between GPUs inside every single node of the system and reduce the total number of MPI data exchanges; (2) We design CUDA kernels instead of using memory copy for copying the fragmented data. These two strategies accelerate the data exchange along the $x$-direction and overcome the problem of the 3D decomposition of MHD simulations on distributed multiGPU systems. Furthermore, since the 3D decomposition is available, it reduces the memory usage for the halo data. More data can be stored and calculated in every GPU, thus improving the overall efficiency. The proposed approach has been developed in an efficient implementation for magnetohydrodynamic (MHD) simulations on distributed multi-GPU systems, called $M G P U-M H D$ code. The code realizes the GPU parallelization of a total variation diminishing (TVD) algorithm for solving the multidimensional ideal MHD equations, extending our work GPU-MHD for single GPU computation [43] to multiple GPUs. Numerical tests and performance measurements are conducted on the TSUBAME 2.0 supercomputer at the Tokyo Institute of Technology. Our code achieves 2 TFLOPS in double precision for the problem with $1200^{3}$ grid points using 216 GPUs. Performance measurements show a significant enhancement with our approach compared to a common 2D decomposition MPI-only implementation.

As future work we plan to use $M G P U-G P U$ for some astrophysical applications, for examples, performing the fast magnetic reconnection [26] and the 3D magnetic Rayleigh-Taylor instability [34]. On the another hand, we plan to implement a module using NVIDIA's IndeX Technology [23] to $M G P U-M H D$ to provide large-scale visualizations while performing the simulation. Due to the data exchange between GPUs on different nodes is still inefficient, we are looking forward to utilizing the new dynamic parallelism and GPU Direct RDMA features of the new Kepler GPUs [22] for further improvements.

\section{Acknowledgments}

The work of Un-Hong Wong was supported by the Japanese Government Scholarship (Research Student) from the Ministry of Education, Culture, Sports, Science and Technology (MEXT) of Japan. The work of Hon-Cheng Wong was supported by the Science and Technology Development Fund of Macao SAR (080/2012/A3). The authors would like to thank the Global Scientific Information and Computing Center, Tokyo Institute of Technology for the use of the resources on the TSUBAME 2.0 supercomputer and Professor Ue-Li Pen at the Canadian Institute for Theoretical Astrophysics, University of Toronto for providing the FORTRAN MHD code. Special thanks to anonymous reviewers for their constructive and valuable comments that helped us to improve the 
paper.

\section{References}

[1] P. Abreu, R. A. Fonseca, J. M. Pereira, and L. O. Silva. PIC codes in new processors: A full relativistic PIC code in CUDA-enabled hardware with direct visualization. IEEE Transactions on Plasma Science, vol. 39, pp. 675-685, 2011.

[2] S. Bastrakov, R. Donchenko, A. Gonoskov, E. Efimenko, A. Malyshev, I. Meyerov, and I. Surmin. Particle-in-cell plasma simulation on heterogeneous cluster systems. Journal of Computational Science, vol. 3, pp. 474-479, 2012.

[3] A. R. Brodtkorb, T. R. Hagen, and M. L. Sætra. Graphics processing unit (GPU) programming strategies and trends in GPU computing. Journal of Parallel and Distributed Computing, vol. 73, pp. 4-13, 2013.

[4] H. Burau, R. Widera, W. Hönig, G. Juckeland, A. Debus, T. Kluge, U. Schramm, T. E. Cowan, R. Sauerbrey, and M. Bussmann. PIConGPU: A fully relativistic particle-in-cell code for a GPU cluster. IEEE Transactions on Plasma Science, vol. 38, pp. 2831-2839, 2010.

[5] V. K. Decyk and T. V. Singh. Adaptable particle-in-cell algorithms for graphical processing units. Computer Physics Communications, vol. 182, pp. 641-648, 2011.

[6] T. Endo, A. Nukada, S. Matsuoka, and N. Maruyama. Linpack evaluation on a supercomputer with heterogeneous accelerators. In Proceedings of the 2010 IEEE International Parallel and Distributed Processing Symposium (IPDPS), pp. 1-8, 2010.

[7] C. R. Evans and J. F. Hawley. Simulation of magnetohydrodynamic flow: a constranined transport method. Astrophysical Journal, vol. 332, pp. 659-677, 1988.

[8] X. Feng, L. Yang, C. Xiang, C. Jiang, X. Ma, S. T. Wu, D. Zhong, and Y. Zhou. Validation of the 3D AMR SIP-CESE solar wind model for four Carrington rotations. Solar Physics, vol. 279, pp. 207-229, 2012.

[9] X. Feng, D. Zhong, C. Xiang, and Y. Zhang. GPU computing in space weather modeling. In ASTRONUM 2012 - the 7th Annual International Conference on Numerical Modeling of Space Plasma Flows, in: ASP Conference Series, vol. 474, pp. 131-139, 2012.

[10] X. Feng, D. Zhong, C. Xiang, and Y. Zhang. GPU-accelerated computing of three-dimensional solar wind background. Science China Earth Sciences, vol. 56, pp. 1864-1880, 2013.

[11] Global Scientific Information and Computing Center, Tokyo Institute of Technology. TSUBAME 2.0 hardware and software specifications. http://www.gsic.titech.ac.jp/sites/default/files/TSUBAME_SPECIFICATIONS_en_0.pdf. 
[12] J. P. Goedbloed and S. Poedts. Principles of Magnetohydrodynamics. Cambridge University Press, 2004.

[13] S. Jin and Z. Xin. The relaxation schemes for systems of conservation laws in arbitrary space dimensions. Communication in Pure and Applied Mathematics, vol. 48, pp. 235-276, 1995.

[14] D. B. Kirk and W. W. Hwu. Programming Massively Parallel Processors. Second Edition, Addison-Wesley, 2013.

[15] X. Kong, M. C. Huang, C. Ren, and V. K. Decyk. Particle-in-cell simulations with charge-conserving current deposition on graphic processing units. Journal of Computational Physics, vol. 230, pp. 1676-1685, 2011.

[16] D. Lee and A. E. Deane. An unsplit staggered mesh scheme for multidimensional magnetohydrodynamics. Journal of Computataion Physics, vol. 228, pp. 952975, 2009.

[17] L. Lin, C. S. Ng, and A. Bhattacharjee. Large-scale high-Lundquist number reduced MHD simulations of the solar corona using GPU accelerated machines. arXiv:1109.6038, 2011.

[18] K. Madduri, E.-J. Im, K. Z. Ibrahim, S. Williams, S. Ethier, and L. Oliker. Gyrokinetic particle-in-cell optimization on emerging multi- and manycore platforms. Parallel Computing, vol. 37, pp. 501-520, 2011.

[19] P. D. Mininni, A. G. Pouquet, and D. C. Montgomery. Small-scale strucutures in three-dimensional magnetohydrnamic turbulence. Physical Review Letters, vol. 97, Article 244503, 2006.

[20] NVIDIA CUDA Zone: http://developer.nvidia.com/category /zone/cuda-zone.

[21] NVIDIA's next generation CUDA compute architecture: Fermi, Whitepaper, V1.1, 2009, http://www.nvidia.com/object/cuda_develop.html.

[22] NVIDIA's next generation CUDA compute architecture: Kepler GK110, Whitepaper, V1.0., 2012. http://www.nvidia.com/object/cuda_develop.html.

[23] NVIDIA's IndeX Technology: http://www.nvidia-arc.com/products/nvidia-index.html.

[24] A. Orszag and C. M. Tang. Small-scale structure of two-dimensional magnetohydrodynamics turbulence. Journal of Fluid Mechanics, vol. 90, pp. 129-143, 1979.

[25] B. Pang, U.-L. Pen, and M. Perrone. Magnetohydrodynamics on heterogeneous architectures: a performance comparison. arXiv:1004.1680, 2010.

[26] B. Pang, U.-L. Pen, and E. T. Vishniac. Fast magnetic reconnection in threedimensional magnetohydrodynamics simulations. Physics of Plasmas, vol. 17, Article 102302, 2010. 
[27] U.-L. Pen, P. Arras, and S. Wong. A free, fast, simple and efficient TVD MHD code. Astrophysical Journal Supplement Series, vol. 149, pp. 447-455, 2003.

[28] T. Shimokawabe, T. Aoki, C. Muroi, J. Ishida, K. Kawano, T. Endo, A. Nukada, N. Maruyama, and S. Matsuoka. An 80-fold speedup, 15.0 TFlops full GPU acceleration of non-hydrostatic weather model ASUCA production code. In Proceedings of the 2010 ACM/IEEE International Conference for High Performance Computing, Networking, Storage and Analysis (SC'10), pp. 1-11, 2010.

[29] T. Shimokawabe, T. Aoki, T. Takaki, A. Yamanaka, A. Nukada, T. Endo, N. Maruyama, and S. Matsuoka. Peta-scale phase-field simulation for dendritic solidification on the TSUBAME 2.0 supercomputer. In Proceedings of the 2011 ACM/IEEE International Conference for High Performance Computing, Networking, Storage and Analysis (SC'11), Article No. 3, 2011.

[30] T. Shimokawabe, T. Aoki, and N. Onodera. A high-productivity framework for multi-GPU computation of mesh-based applications. In Proceedings of the First International Workshop on High-Performance Stencil Computations (HiStencils 2014), pp. 23-30, 2014.

[31] Spherical blast wave test page of Athena3D test suite: http://www. astro.virginia.edu/VITA/ATHENA/blast.html.

[32] G. Stantchev, W. Dorland, and N. Gumerov. Fast parallel-to-grid interpolation for plasma PIC simulations on the GPU. Journal of Parallel and Distributed Computing, vol. 68, pp. 1339-1349, 2008.

[33] G. Stantchev, D. Juba, W. Dorland, and A. Varshney. Using graphics processors for high-performance computation and visualization of plasma turbulence. Computing in Science and Engineering, vol. 11, pp. 52-59, 2009.

[34] J. Stone and T. Gardiner. The magnetic Rayleigh-Taylor instability in three dimensions. The Astrophysical Journal, vol. 671, pp. 1726-1735, 2007.

[35] J. Stone, T. A. Gardiner, P. Teuben, J. F. Hawley, and J. B. Simon. Athena: a new code for astrophysical MHD. Astrophysical Journal Supplement Series, vol. 178 , pp. 137-177, 2008.

[36] G. Strang. On the construction and comparison of difference schemes. SIAM Journal of Numerical Analysis, vol. 5, pp. 506-517, 1968.

[37] Top 500 list: http://www.top500.org.

[38] G. Tóth. The $\nabla \cdot \mathbf{B}=0$ constraint in shock-capturing magnetohydrodynamics codes. Journal of Computational Physics, vol. 161, pp. 605-656, 2000.

[39] R. Ueda, Y. Matsumoto, M. Itagaki, and S.-I. Oikawa. Effectiveness of GPGPU for solving the magnetohydrodynamics equations using the CIP-MOCCT method. Plasma and Fusion Research: Regular Articles, vol. 6, Article 24010192, 2011. 
[40] P. Wang, T. Abel, and R. Kaehler. Adaptive mesh fluid simulations on GPU. New Astronomy, vol. 15, pp. 581-589, 2010.

[41] X. Wang and T. Aoki. Multi-GPU performance of incompressible flow computation by lattice Boltzmann method on GPU cluster. Parallel Computing, vol. 37, pp. 521-535, 2011.

[42] A. Wasiljew and K. Murawski. A new CUDA-based GPU implementation of the two-dimensional Athena code. Bulletin of the Polish Academy of Sciences: Technical Sciences, vol. 61, pp. 239-250, 2013.

[43] H.-C. Wong, U.-H. Wong, X. Feng, and Z. Tang. Efficient magnetohydrodynamic simulations on graphics processing units with CUDA. Computer Physics Communications, vol. 182, pp. 2132-2160, 2011.

[44] U.-H. Wong, H.-C. Wong, and Y. Ma. Global magnetohydrodynamic simulations on multiple GPUs. Computer Physics Communications, vol. 185, pp. 144-152, 2014.

[45] A. Yamanaka, T. Aoki, S. Ogawa, and T. Takaki. GPU-accelerated phasefield simulation of dendritic solidification in a binary alloy. Journal of Crystal Growth, vol. 318, pp. 40-45, 2011.

[46] A. Zachary, A. Malagoli, and P. Colella. A higher-order Godunov method for multidimensional ideal magnetohydrodynamics. SIAM Journal of Scientific Computing, vol. 15, pp. 263-284, 1994.

[47] B. Zink. HORIZON: Accelerated general relativistic magnetohydrodynamics. arXiv:1102.5202, 2011. 